\title{
Article \\ Nature-Inspired Nanoparticles as Paclitaxel Targeted Carrier for the Treatment of HER2-Positive Breast Cancer
}

\author{
Celia Nieto*(D), Milena A. Vega (D) and Eva Martín del Valle *(D) \\ Chemical Engineering Department, Faculty of Chemical Sciences, University of Salamanca, \\ 37008 Salamanca, Spain; mvega@usal.es \\ * Correspondence: celianieto@usal.es (C.N.); emvalle@usal.es (E.M.d.V.); Tel.: +34-923294500 (E.M.d.V.)
}

check for

updates

Citation: Nieto, C.; Vega, M.A.; Martín del Valle, E. Nature-Inspired Nanoparticles as Paclitaxel Targeted Carrier for the Treatment of HER2-Positive Breast Cancer. Cancers 2021, 13, 2526. https://doi.org/ 10.3390/cancers 13112526

Academic Editors: Ana Isabel Torres-Suárez and Ana Isabel Fraguas-Sánchez

Received: 13 April 2021

Accepted: 19 May 2021

Published: 21 May 2021

Publisher's Note: MDPI stays neutral with regard to jurisdictional claims in published maps and institutional affiliations.

Copyright: (C) 2021 by the authors Licensee MDPI, Basel, Switzerland. This article is an open access article distributed under the terms and conditions of the Creative Commons Attribution (CC BY) license (https:/ / creativecommons.org/licenses/by/ $4.0 /)$.
Simple Summary: Great advances have been made in the treatment of an aggressive subtype of breast cancer, known as HER2-positive subtype. However, the antitumor therapy most widely employed in the clinical fight against it has severe side effects, and the apparition of treatment resistances is frequent. To overcome these drawbacks, nano-sized particles had already been developed as targeted vehicles for the drug and the antibody that are usually administered as first-line treatment for this subtype of breast cancer. These nanoparticles showed better results than the drug that they transported. Nevertheless, to further reduce the drug effective dose and its toxicity to normal tissues, other nanoparticles, more advantageous than the previous ones, were developed in the current work. Compared to the previous nanoparticles, those prepared here proved to be more efficacious, and their potential administration may constitute an excellent approach to improve the outcomes of patients suffering from HER2-positive breast cancer.

\begin{abstract}
Despite the advances made in the fight against HER2-positive breast cancer, the need for less toxic therapies and strategies that avoid the apparition of resistances is indisputable. For this reason, a targeted nanovehicle for paclitaxel and trastuzumab, used in the first-line treatment of this subtype of breast cancer, had already been developed in a previous study. It yielded good results in vitro but, with the aim of further reducing paclitaxel effective dose and its side effects, a novel drug delivery system was prepared in this work. Thus, polydopamine nanoparticles, which are gaining popularity in cancer nanomedicine, were novelty loaded with paclitaxel and trastuzumab. The effectiveness and selectivity of the nanoparticles obtained were validated in vitro with different HER2-overexpressing tumor and stromal cell lines. These nanoparticles showed more remarkable antitumor activity than the nanosystem previously designed and, in addition, to affect stromal cell viability rate less than the parent drug. Moreover, loaded polydopamine nanoparticles, which notably increased the number of apoptotic HER2-positive breast cancer cells after treatment, also maintained an efficient antineoplastic effect when validated in tumor spheroids. Thereby, these bioinspired nanoparticles charged with both trastuzumab and paclitaxel may represent an excellent approach to improve current HER2-positive breast cancer therapies.
\end{abstract}

Keywords: breast cancer; HER2-overexpression; drug delivery system; polydopamine nanoparticles; paclitaxel; trastuzumab

\section{Introduction}

Throughout 2020, more than 2.2 million new cases of breast cancer (BC) were diagnosed worldwide and nearly 0.7 million people died from this complex disease, which was the type of cancer with the highest incidence last year [1].

Among the new cases of BC that are diagnosed, around 15-20\% overexpress the human epidermal growth factor receptor-2 (HER2) [2]. HER2, also overexpressed in other types of solid tumors [3,4], can dimerize with other members of its receptor family, and this fact leads to cancer cell proliferation and survival, angiogenesis and metastasis [4]. 
In this way, HER2-overexpression may result in more aggressive clinical behavior and has been linked to worse patient outcomes [2]. However, as the overexpression of this tyrosine kinase (TK) receptor does not occur under normal physiological conditions [3], on the other hand, it has allowed the development of anti-HER2 targeted agents that have dramatically improved the survival rate of patients suffering from HER2-positive (HER2+) BC [2]. Undoubtedly, the most widely used HER2-targeted agent to date has been trastuzumab (Herceptin ${ }^{\circledR}$, Basilea, Switzarland), a humanized monoclonal antibody $(\mathrm{mAb})$ approved by the major drug regulatory agencies for the past two decades for the treatment of both early and metastatic BC [5]. Trastuzumab (Tmab) has been shown to induce tumor regression through different molecular mechanisms and to significantly increase overall survival of patients in multiple trials [6,7]. For this reason, it is nowadays an established part of adjuvant HER2+ BC treatment along with taxanes [6,8]. Nonetheless, Tmab intravenous administration also has some downsides. The two most important ones are its inherent cardiotoxicity and the apparition of acquired Tmab resistances, which cause $15 \%$ of patients to relapse after treatment $[7,9]$.

Likewise, these two drawbacks (severe side effects and apparition of drug resistances) are also characteristic of conventional chemotherapy agents, mainly due to their poor specificity towards neoplastic tissues $[10,11]$. For instance, paclitaxel (PTX), which is frequently administered as adjuvant therapy with Tmab for the treatment of HER2+ BC [12], is very effective in stabilizing the microtubules of cancer cells and triggering subsequent apoptosis. Nevertheless, it has very poor aqueous solubility and low bioavailability, and the PTX formulation that is normally administered in the clinical setting (Taxol ${ }^{\circledR}, \mathrm{NY}$, USA) has severe adverse effects $[13,14]$. Thereby, the targeted delivery of this antimitotic compound is a major necessity $[11,14]$, and it constitutes one of the main goals that nanotechnology is trying to achieve by means of the development of novel drug delivery systems (DDS) [13-15].

With regard to the latter and given that the combination of PTX plus Tmab is regulated and widely employed in the clinical setting, a DDS composed of alginate and piperazine nanoparticles (NPs) was already developed as vehicle for both the mAb and the taxane in a previous work to target HER2+ cancer cells. To enhance PTX aqueous solubility, this drug was previously included in $\beta$-cyclodextrin molecules. The resulting complexes along with Tmab were attached to the mentioned NPs, which were not toxic to human tumor or healthy cells. The nanosystem developed showed greater effectiveness and selectivity than the parent drug [16]. Nonetheless, with the aim of further improving the antitumor activity of the taxane, reducing its effective dose and lowering its adverse effects in an even more remarkable manner, a new DDS for Tmab and PTX was developed in the current work.

This DDS is composed of polydopamine (PDA) NPs, which have acquired great relevance in cancer nanomedicine in recent years thanks to their excellent physicochemical properties $[17,18]$. More specifically, PDA is biocompatible and biodegradable, its chemical versatility permits further functionalization strategies, and it has a strong ability to adsorb drugs via $\pi-\pi$ stacking or hydrogen bond interactions. In addition, PDA NPs are capable of releasing drugs in a pH-responsive manner and [19-21], most importantly, they have proven to have intrinsic antitumor activity $[17,22,23]$.

So far, no studies were found in the literature in which PTX has been loaded to PDA NPs, and since these NPs are capable of charging drugs with high loading rates, this fact was used to directly load PTX to them. Thus, the employment of additional compounds necessary to improve the taxane solubility, like $\beta$-cyclodextrins was avoided by using these NPs, which made the conjugation process more profitable. Furthermore, since PDA NPs have antitumor activity themselves, especially when they are synthesized with 2-propanol [23], synergy between them and PTX was expected.

Otherwise, Tmab was also directly adsorbed in PDA NPs for the first time. In addition, since PDA can be easily functionalized, the mAb was bound by means of the carbodiimide chemistry to try to enhance the receptor-mediated endocytosis and selectivity of the resulting loaded PDA NPs. 
Thereby, two different nanosystems were obtained, whose antitumor activity and neoplastic selectivity were validated by conventional viability assays. These assays were performed with two human HER2+ BC cell lines and with stromal cells. Based on the results obtained, since PDA NPs covalently charged with Tmab had slightly better antitumor effect and reduced the viability of normal cells less, they were chosen to perform further studies. Herein, their cytotoxicity was analyzed by alive/death confocal microscopy (CLSM) assays and, to verify that PTX pharmacological activity did not change upon loading to PDA NPs, NP ability to induce apoptosis in HER2+ cancer cells was verified by flow cytometry. Furthermore, the effectiveness of the loaded PDA NPs was also validated in HER2+ tumor spheroids to prove that it was maintained in these 3D biostructures. For this purpose, the interaction between the spheroids and the loaded PDA NPs was studied by scanning electron microscopy (SEM). Moreover, additional alive/death CLSM assays and cell counting experiments were carried out. As a result, it was proven that PDA NPs loaded with both PTX and Tmab, were able to significantly reduce the survival rate of HER2+ malignant cells cultured in both 2D and 3D, to a similar or even greater extent than equivalent concentrations of free PTX. Moreover, loaded PDA NPs were notably less toxic to stromal cells than the parent drug. Therefore, their employment may constitute a good strategy to improve PTX bioavailability, while reducing its effective dose and its adverse effects. Likewise, avoiding the apparition of treatment resistances may be possible with the administration of these targeted PDA NPs, which proved to be more advantageous than the previous, similarly reported paclitaxel and trastuzumab DDS.

\section{Results}

\subsection{Synthesis and Characterization of PDA NPS}

Initially, PDA NPs were prepared by the oxidation and self-polymerization of dopamine in a basic, aqueous medium containing 2-propanol (2-PrOH) and $\mathrm{NH}_{4} \mathrm{OH}$. Even though ethanol is the alcohol normally used in this synthesis procedure $[17,23,24], 2-\mathrm{PrOH}$ was chosen because it had been found in a previous work that its employment also allowed PDA NPs to be obtained, and that it conferred them greater antitumor activity in comparison to other alcohols [23]. Otherwise, the $\mathrm{NH}_{4} \mathrm{OH}$ concentration was adjusted in order to prepare NPs with a diameter smaller than $200 \mathrm{~nm}$, since PDA NPs with that size had been observed to have greater inherent antineoplastic effect than bigger ones [22].

Once obtained, PDA NP hydrodynamic diameter was determined by dynamic light scattering (DLS). It was found to be $179.3 \pm 41 \mathrm{~nm}(\mathrm{PDI}=0.07)$ (Figure 1a). In addition, PDA NP surface charge (zeta potential), which was-27.4 $\pm 1.5 \mathrm{mV}$, was also determined, and PDA NPs were characterized by transmission electron microscopy (TEM) to analyze their morphology and to obtain a size-range histogram (Figure 1b). According to this, NPs had an average diameter of $124.3 \pm 19.2 \mathrm{~nm}$, which was a lower value than that observed by DLS possibly due to the dehydration suffered by the NPs when preparing TEM samples [25].

(a)

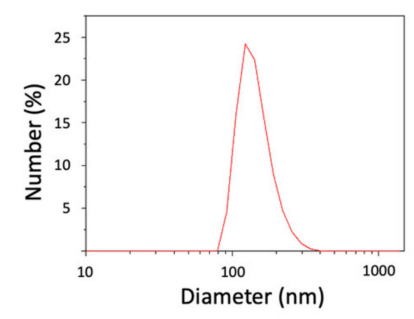

(b)

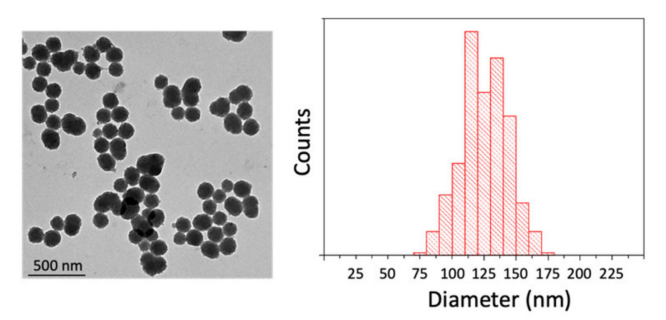

Figure 1. (a) DLS number distribution of the PDA NPs synthesized, suspended in Trizma base solution ( $\mathrm{pH}$ 10.0); (b) TEM image and size-range histogram of the PDA NPs obtained. 


\subsection{PDA NP Loading with PTX and Tmab}

Next, synthesized PDA NPs were washed through five centrifugation-redispersion cycles in phosphate buffer saline (PBS) and charged with PTX and Tmab following two different methods (Figure 2). First, the drug and the antibody were loaded to PDA NPs through a simple chemisorption process (PDA NPs@Tmab@PTX) by adding solutions of both compounds to PDA NP suspensions. Secondly, the carbodiimide coupling strategy was used to covalently bind Tmab to PDA NPs with the aim of verifying whether this type of conjugation improved the antineoplastic activity and selectivity of the resulting NPs (PDA NPs•Tmab@PTX). To do this, in order for the carbodiimide chemistry to be more efficient, the $\mathrm{pH}$ of the NP suspensions was acidified [26], and carboxyl groups of PDA NP surface were activated with EDC and NHS solutions [5]. Later, in two sequential steps, Tmab and PTX solutions with a similar concentration to those used in the adsorption process were incorporated into NP suspensions.

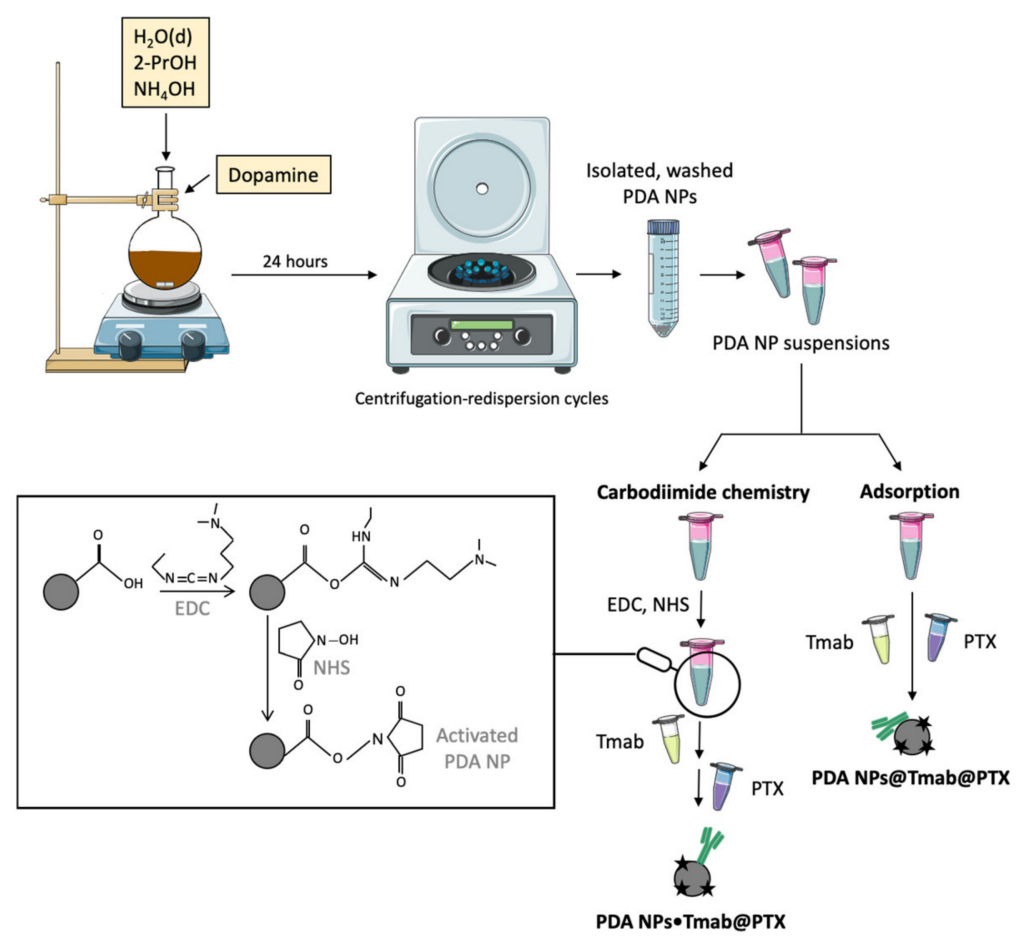

Figure 2. Schematic representation of PDA NPs synthesis and loading with PTX and Tmab by following two different methods in the latter case: adsorption and the carbodiimide coupling chemistry $\left(\mathrm{H}_{2} \mathrm{O}(\mathrm{d})\right.$-Deionized water; EDC-N-(3-Dimethylaminopropyl)- $\mathrm{N}^{\prime}$-ethylcarbodiimide hydrochloride; NHS-N-hydroxysuccinimide), PDA-Polydopamine.

Charged PDA NPs were isolated by centrifugation, and Tmab and PTX loading efficiencies were both determined by difference, measuring the absorbance of the supernatants obtained by UV-Vis spectrophotometry. Results were obtained according to the two different Tmab conjugation strategies followed and are summarized in Table 1. As can be seen, there were no very significant differences between PTX loading efficiency values, but Tmab conjugation efficiency was greater when the carbodiimide chemistry was employed.

Moreover, zeta potential of PDA NPs@Tmab@PTX and PDA NPs•Tmab@PTX was also analyzed after the conjugation processes. It can be consulted in the Supplementary Material (Table S1). 
Table 1. Conjugation efficiencies (\%) and Tmab (Trastuzumab) and PTX (Paclitaxel) content in PDA $\mathrm{NPs}(\mu \mathrm{g} / \mathrm{mg})$ after adsorbing the drug and loading the antibody by adsorption or by means of the carbodiimide chemistry.

\begin{tabular}{ccccc}
\hline & \multicolumn{2}{c}{ Adsorption } & \multicolumn{2}{c}{ Carbodiimide Chemistry } \\
\cline { 2 - 5 } & $\begin{array}{c}\text { Conjugation } \\
\text { efficiency }\end{array}$ & Drug/Antibody & Conjugation & Drug/Antibody \\
& $27.4 \%$ & content & efficiency & content \\
Tmab & $18.4 \%$ & $2.3 \mu \mathrm{g} / \mathrm{mg} \mathrm{NPs}$ & $37.8 \%$ & $1.9 \mu \mathrm{mg} \mathrm{NPs}$ \\
PTX & $19.1 \%$ & $2.44 \mu \mathrm{mg} / \mathrm{mg}$ NPs \\
\hline
\end{tabular}

\subsection{In Vitro Evaluation of the Antitumor Activity and Selectivity of PDA NPs Charged with} Tmab and PTX

In order to evaluate and compare the antitumor activity and selectivity of the two types of loaded PDA NPs obtained, MTT assays were carried out with two breast carcinoma cell lines overexpressing HER2 (BT474 and SKBR3) and with stromal cells (HS5 cell line) [16].

For such assays, all cells were treated with 0.035 and $0.042 \mathrm{mg} / \mathrm{mL}$ PDA NP concentrations. As already mentioned, these NPs have intrinsic antineoplastic activity, and it had been proven that these two concentrations were able to reduce tumor cell viability in a remarkable manner in previous studies [23]. Likewise, the same concentrations of PDA NPs charged only with PTX or/and Tmab were administered, and cells were also treated with concentrations of the drug and antibody equivalent to those loaded to PDA NPs. To perform the viability assays, a previously developed protocol that avoids overestimating cell viability by subtracting PDA contribution to the absorbance values of the samples was followed [17]. Results obtained after cell treatment with PDA NPs in which Tmab was adsorbed can be found in Figure S1, while those obtained after the administration of PDA NPs covalently conjugated with Tmab are summarized in Figure 3.

As can be seen in both figures, bare PDA NPs were not excessively toxic to normal cells, but they notably reduced BC cell survival rate. These results were in line with those obtained in prior research, in which it had been hypothesized that the great affinity of PDA NPs for the iron (III) existing in the lysosomes could be responsible for their cytotoxicity, which could be thus potentially related to a ferroptosis-mediated excessive production of reactive oxygen species (ROS) in tumor cells [22-24].

Otherwise, both PDA NPs•Tmab@PTX and PDA NPs@Tmab@PTX were found to be highly effective, since they reduced the viability of HER2+ BC cells to a slightly greater extent than free PTX concentrations equivalent to those adsorbed in most of the conditions studied $(p<0.05)$ (Tables S2-S4). In this manner, when BT474 cells were treated with PDA NPs•Tmab@PTX, only 10-17\% of them survived after $72 \mathrm{~h}$, while this percentage was 13-19\% when PDA NPs@Tmab@PTX were employed. Regarding the viability of the SKBR3 cell line, it was 10-14\% $72 \mathrm{~h}$ after treatment with PDA NPs•Tmab@PTX while, when PDA NPs@Tmab@PTX were administered, SKBR3 survival rate was close to 15-23\%. Furthermore, it was noticed in both cases that Tmab covalent conjugation or adsorption to PDA NPs enhanced their antineoplastic activity, since it was greater when NPs were simultaneously charged with the antibody and the drug than when they were only loaded with PTX. Thus, PDA NPs•Tmab@PTX and PDA NPs@Tmab@PTX reduced BT474 and SKBR3 cell viability rates between 5-15\% more than PDA NPs@PTX (depending on the NP concentration and treatment time), a fact that revealed that Tmab loading could enhance NP endocytosis in HER2+ tumor cells.

On the contrary, Tmab loading to PDA NPs made them less toxic to stromal cells than PDA NPs charged only with PTX. Thereby, when HS5 cells were treated with PDA NPs•Tmab@PTX and PDA NPs@Tmab@PTX, their survival rate was 23-30\% higher than when the different concentrations of PDA NPs@PTX were used. With respect to the free parent drug, this gap was 25-34\% and, compared to BC cells, about 26-36\% more stromal cells survived after the same treatment. In fact, Tmab loading even slightly improved the intrinsic toxicity of bare PDA NPs to normal cells. 
(a)

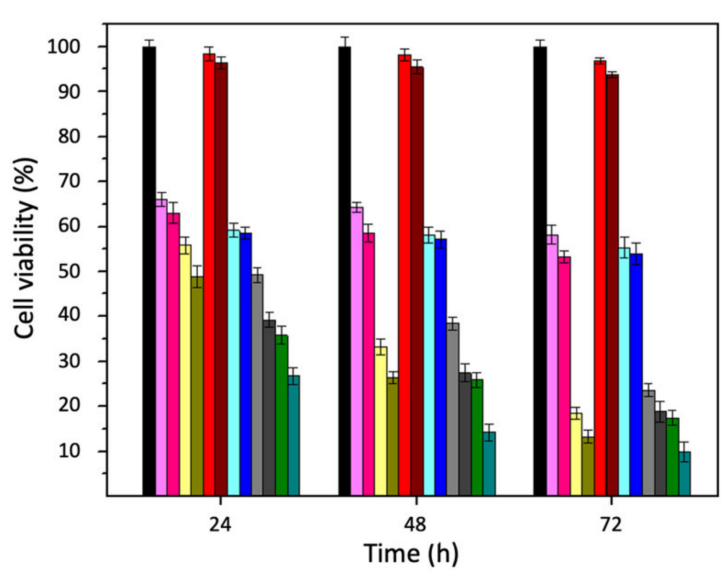

(b)

SKBR3 cell line

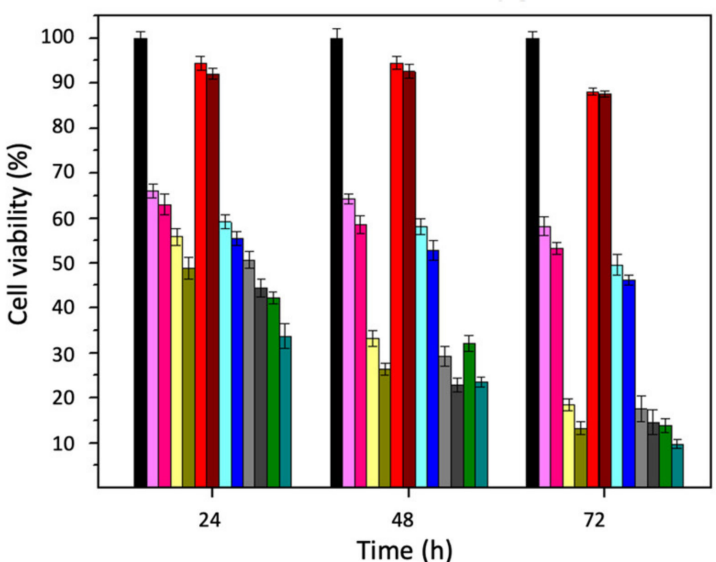

Control $\square$ PDA NPs $(0.035 \mathrm{mg} / \mathrm{mL}) \quad \square$ PDA NPs $(0.042 \mathrm{mg} / \mathrm{mL}) \square \operatorname{PTX}(99.5 \mathrm{nM}) \quad \square \operatorname{PTX}(119.5 \mathrm{nM}) \square \operatorname{Tmab}(0.45 \mathrm{nM})$

Tmab (0.54 nM) $\square$ PDA NPs•Tmab $(0.035 \mathrm{mg} / \mathrm{mL}) \square$ PDA NPs $\bullet T m a b(0.042 \mathrm{mg} / \mathrm{mL}) \quad \square$ PDA NPs@PTX $(0.035 \mathrm{mg} / \mathrm{mL})$

$\square$ PDA NPs@PTX (0.042 mg/mL) $\square$ PDA NPs•Tmab@PTX (0.035 mg/mL) $\square$ PDA NPs•Tmab@PTX (0.042 mg/mL)

(c)

HS5 cell line

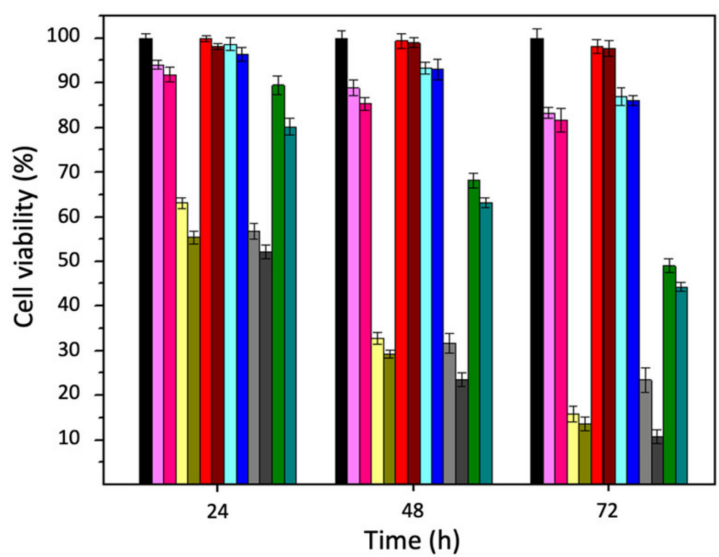

Figure 3. Survival rates of BT474 (a), SKBR3 (b) and HS5 (c) cells after treatment with 0.035 and $0.042 \mathrm{mg} / \mathrm{mL}$ bare PDA NPs, PDA NPs•Tmab, PDA NPs@PTX and PDA NPs•Tmab@PTX, as well as with concentrations of free Tmab and PTX similar to those loaded to PDA NPs.

Even though the differences in terms of efficacy and selectivity between PDA NPs@ Tmab@PTX and PDA NPs•Tmab@PTX were not considered statistically significant $(p>0.05)$ (Tables S5-S7), those NPs that had the antibody covalently bound decreased BT474 and SKBR3 viability by $1.2-13.5 \%$ more (depending on concentration and time) than PDA NPs@Tmab@PTX. Likewise, PDA NPs•Tmab@PTX affected HS5 survival rate by 2.2-15.9\% less than PDA NPs@Tmab@PTX. For this reason, PDA NPs•Tmab@PTX were chosen to perform further studies.

Herein, their antineoplastic activity was also validated by alive/death CLSM assays, which were performed with the BT474 cell line. These HER2-overexpressing cells were treated with $0.035 \mathrm{mg} / \mathrm{mL}$ PDA NPs•Tmab@PTX and with a concentration of free PTX equivalent to that adsorbed to compare the results. Different CLSM images were acquired once 48 and $72 \mathrm{~h}$ elapsed. These images have been included in Figure $4 \mathrm{a}$ and, from them, the average percentage of living and dead cells was determined for each treatment condition. While 48 and $72 \mathrm{~h}$ after treatment with free PTX $36.1 \%$ and $16.7 \%$ of BT474 cells remained alive, $36.3 \%$ and $14.3 \%$ of them survived when treated with $0.035 \mathrm{mg} / \mathrm{mL}$ 
PDA NPs•Tmab@PTX for 48 and $72 \mathrm{~h}$ respectively. Thereby, results of these CLSM assays proved that PDA NPs•Tmab@PTX were highly effective.

Finally, given that PTX exerts its pharmacological activity by inducing apoptosis of tumor cells [14], the number of early and late apoptotic BT474 cells was determined by flow cytometry $48 \mathrm{~h}$ after treatment with PDA NPs•Tmab@PTX $(0.035 \mathrm{mg} / \mathrm{mL})$ and free PTX $(99.5 \mathrm{nM})$. The aim was to verify that the taxane was mainly responsible for the antineoplastic effect of the loaded PDA NPs, and that its adsorption and the later Tmab conjugation to PDA NPs had not altered its antitumor activity. A FITC Annexin V apoptosis detection kit with 7AAD was used [27]. It was observed that treatment with PDA NPs•Tmab@PTX increased the number of early apoptotic HER2+ breast tumor cells $\left(7 \mathrm{AAD}^{-} /\right.$Annexin $\left.\mathrm{V}^{+}\right)$in a similar manner than treatment with an equivalent concentration of free PTX (Figure 4b). Therefore, PTX pharmacological activity was not significantly reduced upon loading to PDA NPs.

(a)
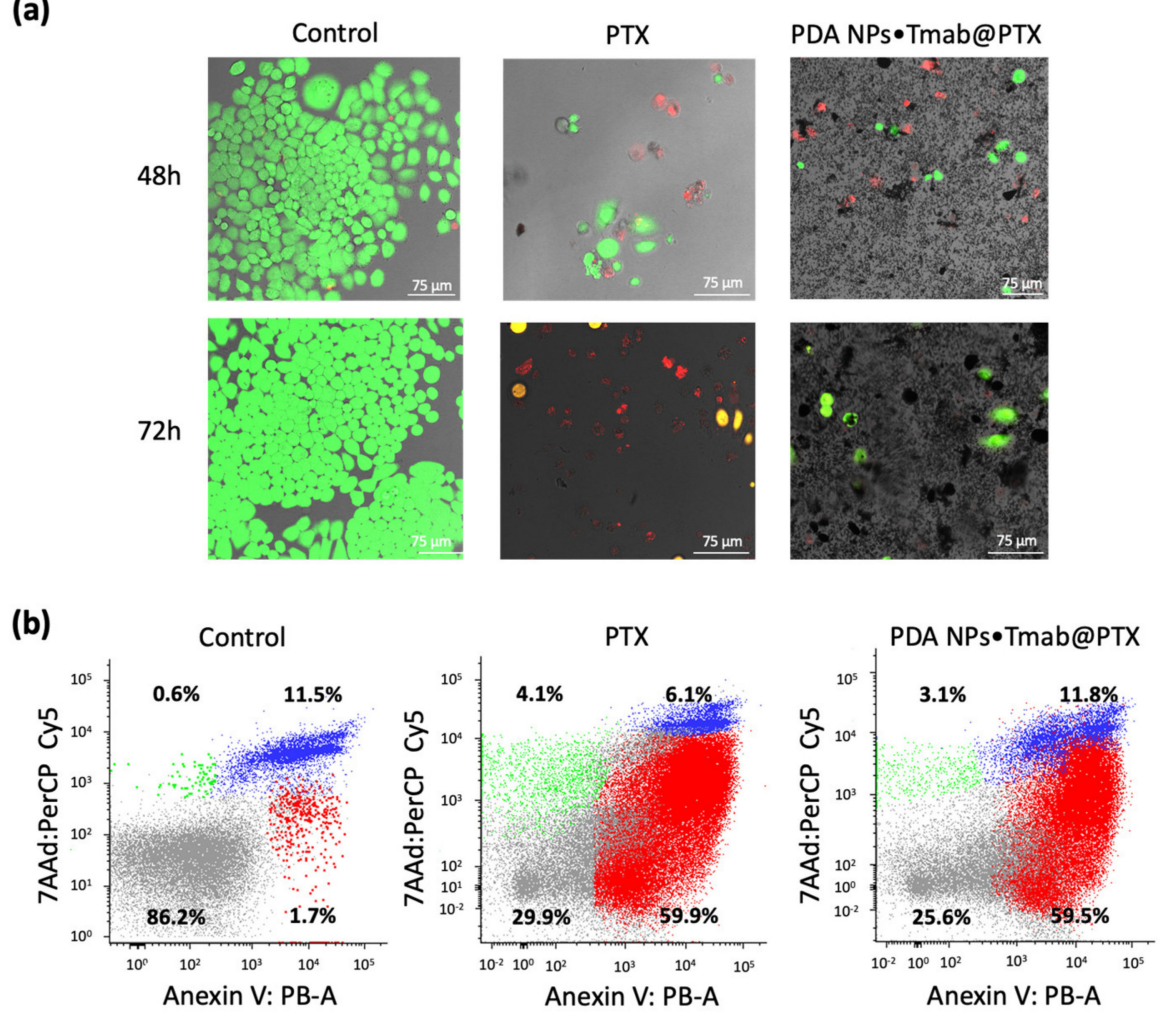

Figure 4. (a) CLSM images of BT474 cells 48 and $72 \mathrm{~h}$ after treatment with PTX (99.5 nM) and PDA NPs•Tmab@PTX $(0.035 \mathrm{mg} / \mathrm{mL})$. Cell viability and death were assessed by using calcein AM (green) and propidium iodide (red), respectively; (b) flow cytometry analysis showing the percentage of apoptotic and necrotic BT474 cells $48 \mathrm{~h}$ after treatment with PTX (99.5 nM) and PDA NPs•Tmab@PTX $(0.035 \mathrm{mg} / \mathrm{mL})$. The vertical axis indicates the cells labeled with 7AAD (PerCP) and the horizontal axis indicates those stained by Annexin V (FITC) (early apoptosis: right lower, late apoptosis/necrosis: right upper).

\subsection{Assessment of the Antitumor Effect of PDA NPs Loaded with Tmab and PTX in $3 D$ Cell Cultures}

Since in vitro 3D culture systems mimic the in vivo growth conditions of solid tumors better than conventional monolayer cells cultures [28], they have been largely adopted as a model for drug and nanomedicine screening [29,30]. Among the numerous spherical cancer models that can be developed, multicellular tumor spheroids (MCTS), which are obtained by growing cancer cell lines under non-adherent conditions, have been reported to accurately mimic the drug sensitivity behavior of in vivo tumors [29,31]. For this reason, 
in the current work, developing MCTS derived from the BT474 cell line was decided to further evaluate the antitumor efficacy of the PDA NPs•Tmab@PTX.

Thus, BT474 cells were assembled in a 3D architecture after seeding them in U-shapedbottom microplates. By day 3 of growth, they had formed compact multicellular spheres about $1 \mathrm{~mm}$ in diameter. At that time, they were treated with PDA NPs•Tmab@PTX $(0.035 \mathrm{mg} / \mathrm{mL})$ and with free PTX in an equivalent concentration to that adsorbed to PDA NPs. Three different types of studies were performed.

First, with the aim of analyzing the interaction between the loaded PDA NPs and the 3D cell biostructures, SEM was employed to acquire images of spheroid sections in a secondary electron mode after critical point drying [30]. These images were taken 48 and $72 \mathrm{~h}$ after treatment. Morphological differences between the control spheroids and the spheroids treated with both free PTX and loaded PDA NPs could be observed, especially when $72 \mathrm{~h}$ had elapsed. In this way, after treatment with the taxane, BT474 spheroids became smaller and disaggregated. This reduction in size and the structural disorganization with loss of integrity could already be detected after exposure to PDA NPs•Tmab@PTX, with which spheroids acquired a grape bunch-shape in which individual cells could be better distinguished (Figure 5). Moreover, in both the images obtained from the spheroids treated with free PTX and with PDA NPs•Tmab@PTX, filamentous-like structures were observed that might correspond to PTX crystals formed in solution [32].

(a)
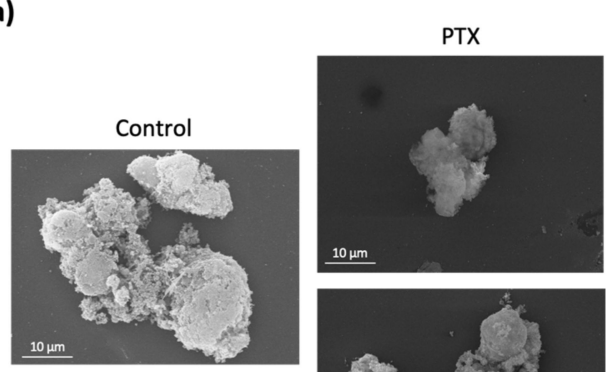

PDA NPs•Tmab@PTX
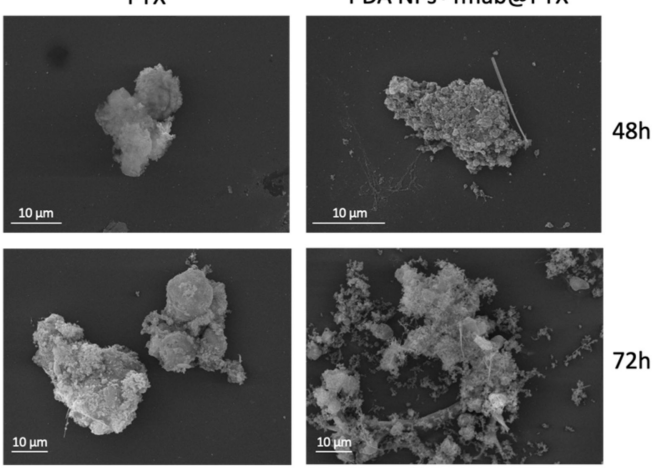

(b)

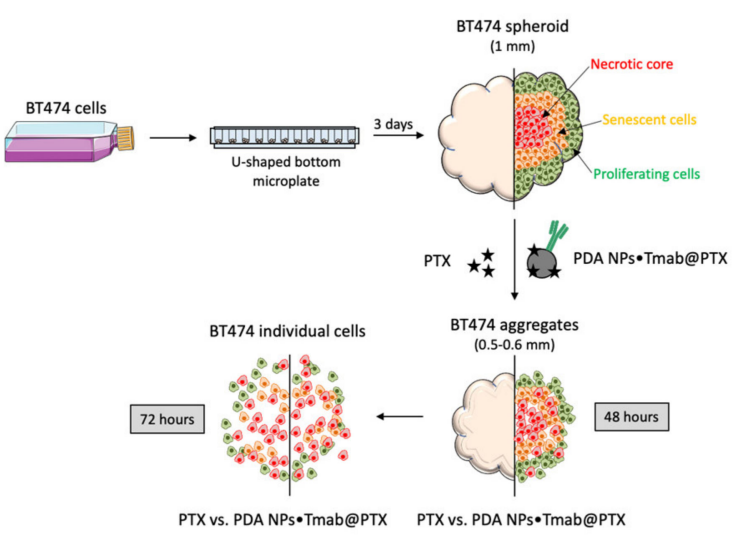

Figure 5. (a) SEM characterization of sections of BT474 spheroids 48 and $72 \mathrm{~h}$ after treatment with PTX (99.5 nM) and PDA NPs•Tmab@PTX (0.035 mg/mL); (b) schematic representation of how BT747 spheroids were developed and the effect that PTX vs. PDA NPs•Tmab@PTX treatment had on their structure and morphology.

Second, additional live/death CLSM assays were also carried out with the 3D cell cultures (Figure 6a). Again, confocal images were taken 48 and $72 \mathrm{~h}$ after spheroid treatment, just as with the monolayer cell cultures. Control images allowed an appreciation of how BT474 MCTS had a necrotic core surrounded by a viable rim, with an inner layer of quiescent cells and an outer layer of proliferating cells. This phenomenon occurs in large tumor 
spheroids $(>500 \mu \mathrm{m})$ [29]. Otherwise, the results obtained by SEM were corroborated with these assays, since it could be noticed that both free PTX and PDA NPs•Tmab@PTX, were able to reduce the size of the spheroids developed and to disaggregate them, especially after $72 \mathrm{~h}$.

Likewise, the effect of PDA NPs•Tmab@PTX treatment on spheroid morphology was borne out by phase-contrast images, which can be found in the Supplementary Material (Figure S2). From them, it could be observed how after $48 \mathrm{~h}$, loaded PDA NPs made the spheroids smaller and decreased their density. In addition, their margins were less marked, an indication of the cellular disassemble that was beginning to take place.

Lastly, the viability of the BT474 cells that made up the spheroids was determined by cell-counting $48 \mathrm{~h}$ after treating them with free PTX and PDA NPs•Tmab@PTX, as well as with $0.035 \mathrm{mg} / \mathrm{mL}$ bare PDA NPs. Results obtained can be found in Figure $6 \mathrm{~b}$. As can be seen, the viability of the control spheroids was close to $71 \pm 4 \%$ due to the existence of the necrotic core described in the previous paragraph, caused by the diffusion gradient of oxygen and nutrients [29]. The mean survival rate of the spheroids treated with unloaded PDA NPs was about $65 \pm 5 \%$ while, when free PTX and PDA NPs•Tmab@PTX were administered, their viability was reduced to $58 \pm 5 \%$ and $59 \pm 6 \%$, respectively. Therefore, it was proven that loaded PDA NPs were not only effective in inducing apoptosis of monolayer HER2+ BC cells. When these cells were forming 3D structures, they also showed a cytotoxicity similar to that of the parent drug, even spreading worse [33].

(a) Control

$48 \mathrm{~h}$

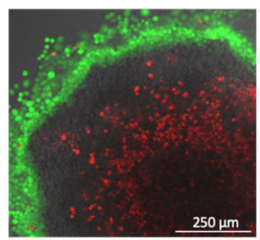

$72 \mathrm{~h}$

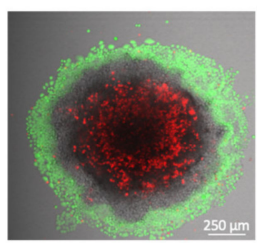

PTX
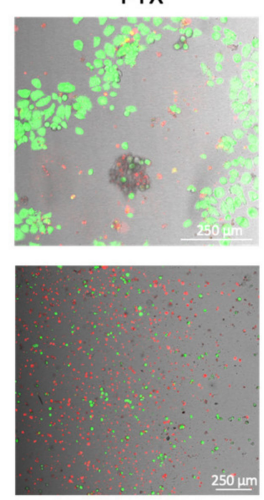

(b)
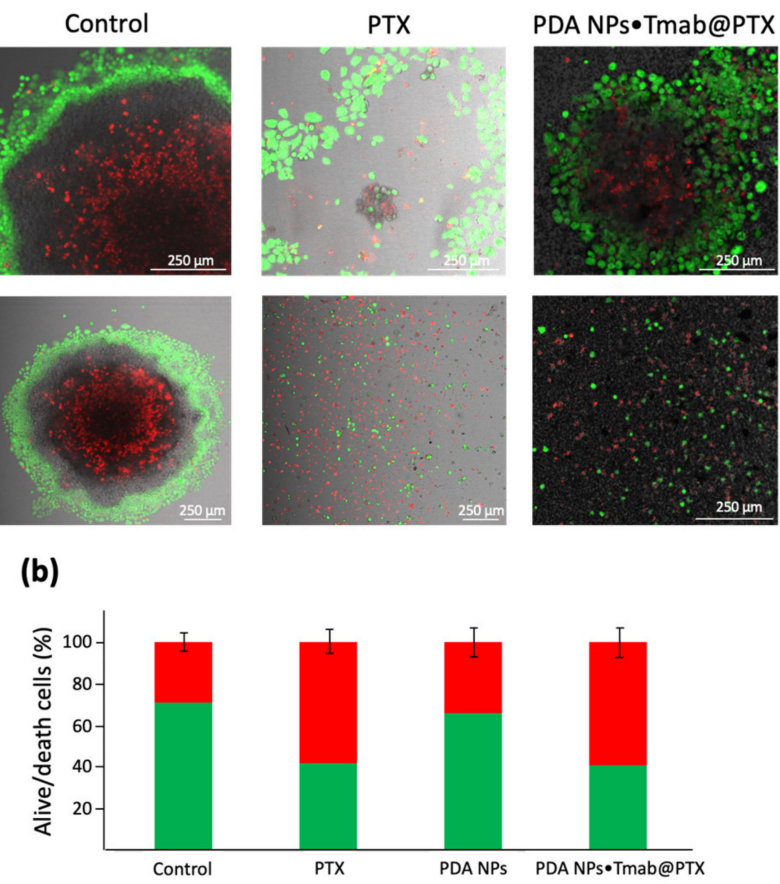

Figure 6. (a) CLSM images of BT474 MCTS 48 and $72 \mathrm{~h}$ after treatment with PTX (99.5 nM) and PDA NPs•Tmab@PTX $(0.035 \mathrm{mg} / \mathrm{mL})$. Cell viability and death were again assessed by using calcein AM (green) and propidium iodide (red); (b) percentage of living (green) and dead (red) BT474 cells cultured in 3D spheres, determined by cell-counting, $48 \mathrm{~h}$ after treatment with PTX (99.5 nM), PDA NPs $(0.035 \mathrm{mg} / \mathrm{mL})$ and PDA NPs•Tmab@PTX $(0.035 \mathrm{mg} / \mathrm{mL})$.

\section{Discussion}

In the last couple of decades, nanotechnology, taking advantage of the deeper knowledge that the scientific community has acquired about cancer biology, has become essential for the development of DDS that seek to overcome the drawbacks of conventional chemotherapy $[10,11,15]$. Thus, for instance, with the aim of reducing Tmab and PTX side effects while potentially avoiding the apparition of resistances to these agents, we had 
already designed alginate and piperazine NPs as a targeted nanovehicle for the anti-HER2 $\mathrm{mAb}$ and the taxane [16]. In a similar manner, other authors developed targeted micelles to transport Tmab and PTX to improve HER2+ BC treatment [33]. In both cases, the polymeric NPs and the micelles were inactive, so they did not show cytotoxicity. Moreover, including PTX into $\beta$-cyclodextrins was necessary before loading it to alginate-piperazine nanoparticles, and encapsuling the taxane into the micelles took a two -day procedure [16,34]. Thus, in order to make the PTX loading process easier and more profitable, and further reduce the effective doses and the side effects of this drug, a novel PTX-Tmab targeted DDS was developed in the current study to improve HER2+ BC treatment.

This novel nanovehicle was made up of PDA NPs, which have specific antineoplastic activity themselves $[17,22,23]$, can directly adsorb drugs with great loading rates [19], and can release these drugs in a $\mathrm{pH}$-sensitive manner $[35,36]$. Thanks to these three properties, it was expected that the taxane could be directly loaded to the aforementioned NPs, that synergy could occur between PDA NPs and PTX, and that PDA pH-sensitivity could improve the selectivity of the DDS obtained, enhancing that provided by Tmab binding.

Herein, PDA NPs (approximately $180 \mathrm{~nm}$ in size) were prepared and easily loaded with PTX for the first time. Likewise, the nanosystem was novelty decorated by Tmab, which was both adsorbed and covalently bound by means of the carbodiimide chemistry. Two types of loaded PDA NPs were obtained, PDA NPs•Tmab@PTX and PDA NPs@Tmab@PTX, which were shown to be highly effective in vitro, reducing the viability rate of HER2+ BC cells similarly or further than PTX concentrations equivalent to those adsorbed. In addition, compared to the other Tmab-PTX-transporting nanosystems mentioned previously [16,34], loaded PDA NPs were found to have more remarkable antitumor activity, perhaps because their inherent toxicity was added to PTX pharmacological activity. In this manner, PDA NPs loaded with both Tmab and PTX reduced the viability of HER2+ breast tumor cells to a greater or equal extent than the similar reported nanomedicines [16,34], but transporting a smaller amount of PTX and having been administered in a lower concentration to the cells. In addition, loaded PDA NPs were not only effective when validated in monolayer cell cultures, but they were also capable of disintegrating and reducing the viability survival rate of HER2+ breast tumor spheroids.

Otherwise, apart from being effective, charged PDA NPs, which were proven to induce apoptosis in target HER2+ BC cells, reduced stromal cell viability to a lesser extent than similar PTX concentrations. Thus, stromal cell survival rate was notably less affected than that of breast tumor cells upon treatment with loaded PDA NPs, while equivalent PTX concentrations reduced the viability of both types of cells in a similar way.

Therefore, PTX loading to PDA NPs also carrying Tmab could constitute a good strategy to improve the bioavailability of the drug in an efficient manner while reducing its adverse effects. Furthermore, their administration may even avoid the apparition of potential resistances by targeting HER2+ tumor cells thanks to Tmab presence, as well as by releasing PTX in a pH-responsive way in neoplastic tissues.

\section{Materials and Methods}

\subsection{Chemicals}

Dopamine hydrochloride, $\mathrm{NH}_{4} \mathrm{OH}(28-30 \%)$, $\mathrm{PBS}$ (0.01 M, pH 7.4), N-hydroxysuccinimide (NHS), N-(3-Dimethylaminopropyl)-N'-ethylcarbodiimide hydrochloride (EDC), PTX (from semisynthetic, $>97 \%$ ), Dulbecco's modified Eagle's medium (DMEM), fetal bovine serum (FBS, USA origin), thiazolyl blue tetrazolium bromide (MTT reagent) and dimethyl sulfoxide (DMSO) were all supplied by Sigma-Aldrich (Darmstadt, Germany). Penicillinstreptomycin $(5000 \mathrm{U} / \mathrm{mL})$, calcein $\mathrm{AM}$, propidium iodide ReadyProbes ${ }^{\mathrm{TM}}$ reagent, Gibco ${ }^{\mathrm{TM}}$ Trypsin-EDTA $(0.25 \%)$ and trypan blue stain $(0.4 \%)$ were obtained from Thermo Fisher Scientific (Eugene, OR, USA). 2-PrOH and $\mathrm{HCl}(37 \%)$ were purchased from PanReac Química S.L.U. (Castellar del Vallès, Barcelona, Spain). Tmab was gifted from the Instituto de Biología Molecular y Celular del Cáncer (Salamanca, Spain) and the FITC Annexin V apoptosis detection kit with 7AAD was obtained from Immunostep (Salamanca, Spain). 


\subsection{Synthesis and Characterization of PDA NPS}

PDA NPs about $180 \mathrm{~nm}$ in size were prepared by dopamine oxidation and selfpolymerization in an alkaline medium. Briefly, an ammonia aqueous solution $(2.5 \mathrm{~mL})$ was mixed with 2- $\mathrm{PrOH}(40 \mathrm{~mL})$ and $\mathrm{H}_{2} \mathrm{O}(\mathrm{d})(90 \mathrm{~mL})$ under magnetic stirring at room temperature for $30 \mathrm{~min}$. Dopamine hydrochloride $(0.5 \mathrm{~g})$ was dissolved in $\mathrm{H}_{2} \mathrm{O}(\mathrm{d})(10 \mathrm{~mL})$ and added to the above solution [23]. The resulting mixture was left to react for $24 \mathrm{~h}$ and PDA NPs obtained were isolated by centrifugation. Four centrifugation-redispersion cycles in $\mathrm{H}_{2} \mathrm{O}(\mathrm{d})$ were performed to eliminate any residue, and PDA NPs were finally resuspended in $\mathrm{H}_{2} \mathrm{O}(\mathrm{d})$ in a $2 \mathrm{mg} / \mathrm{mL}$ concentration once washed.

Next, to characterize PDA NPs, their hydrodynamic diameter was determined by DLS on the basis of their intensity-average size distribution, along with their zeta potential (Zetasizer Nano ZS90, Malvern Instruments Inc., Royston, Hertfordshire, UK). For such a purpose, PDA NPs were dispersed in Trizma base solution ( $\mathrm{pH} 10.0)$ in a concentration lower than $0.01 \%$ (WT). Moreover, PDA NP size and morphology were also characterized by TEM (Tecnai Spirit Twin, Fei Company, Hillsboro, OR, USA). PDA NPs were again resuspended in $\mathrm{H}_{2} \mathrm{O}$ (d) in a concentration inferior to $0.01 \%$ (WT) to prepare the samples and drops of this dispersion were deposited on copper grids with a collodion membrane. NPs were allowed to dry for $24 \mathrm{~h}$ and TEM images of at least 300 different PDA NPs were taken with a $120 \mathrm{kV}$ voltage acceleration. To conclude, these images were analyzed (ImageJ software, NIH, Bethesda, MD, USA) to make a NP size-range histogram.

\subsection{PDA NP Loading with Tmab and/or PTX}

In order to subsequently carry out in vitro studies, PDA NPs were washed through five more centrifugation-redispersion cycles in PBS before being loaded with Tmab and PTX. Next, this loading process was performed following two different methods. On one hand, to adsorb both Tmab and PTX, solutions of the antibody $(1.35 \mu \mathrm{M}, 17.5 \mu \mathrm{L})$ and the drug $\left(0.52 \mu \mathrm{M}, 20.2 \mu \mathrm{L}, 2: 1 \mathrm{H}_{2} \mathrm{O}(\mathrm{d}) / \mathrm{DMSO}\right)$ were incorporated to suspensions of PDA NPs $(2 \mathrm{mg} / \mathrm{mL}, 350 \mu \mathrm{L})$. The resulting mixtures were kept under orbital shaking (100 rpm) in dark conditions, and loaded PDA NPs were isolated the next day by centrifugation [37].

On the other hand, to covalently attach Tmab and adsorb PTX to PDA NPs, the $\mathrm{pH}$ of the NP suspensions $(2 \mathrm{mg} / \mathrm{mL}, 1 \mathrm{~mL})$ was adjusted to $4.7-4.8$ by dropping $\mathrm{HCl}$ $(37 \%)$. Then, solutions of EDC $(193 \mathrm{mg} / \mathrm{mL}, 10 \mu \mathrm{L})$ and NHS $(58 \mathrm{mg} / \mathrm{mL}, 10 \mu \mathrm{L})$ were added. NPs were kept under orbital shaking (100 rpm) for $40 \mathrm{~min}$ and, after that time, a Tmab solution $(1.35 \mu \mathrm{M}, 50 \mu \mathrm{L})$ was incorporated into the suspensions. Mixtures were kept under agitation for 3 additional hours and, finally, a PTX solution $(0.52 \mu \mathrm{M}, 58 \mu \mathrm{L}$, 2:1 $\mathrm{H}_{2} \mathrm{O}(\mathrm{d}) / \mathrm{DMSO}$ ) was also added. Final mixtures were left shaking overnight in dark conditions, and charged PDA NPs were isolated by centrifugation, too [16].

The same procedures were carried out to prepare PDA NPs@Tmab, PDA NPs•Tmab and PDA NPs@PTX but, in these cases, only the solution of the antibody or the drug were incorporated to the NP suspensions. All supernatants were preserved to determine Tmab and PTX loading efficiencies and content in PDA NPs.

\subsection{Determination of Tmab and PTX Loading Efficiencies and Content}

While quantification of PTX in PDA NP supernatants was achieved via spectrophotometry at $227 \mathrm{~nm}$ (UV-1800, Shimadzu Corporation, Kioto, Japan), Tmab concentration in them was determined using the Pierce ${ }^{\mathrm{TM}} \mathrm{BCA}$ Protein Assay Kit (Thermo Fisher Scientific, Eugene, OR, USA) following the instructions detailed by the manufacturer. The amount of Tmab and PTX charged in the NPs was determined by difference, and the different loading efficiencies (\%) and the antibody and drug content in the charged PDA NPs were found by applying the following equations [38]:

$$
\text { Loading ef ficiency }(\%)=\frac{\text { Weight of antibody or drug found loaded }}{\text { Weight of total antibody or drug used }} \times 100
$$




$$
\text { Antibody or drug content }(W / W)=\frac{\text { Weight of antibody or drug found loaded }}{\text { Weight of loaded PDA NPS }}
$$

\subsection{Cell Culture}

BT474, SKBR3 and HS5 cell lines (ATCC, Wessel, Germany) were cultured as instructed. They were grown in DMEM, supplemented with FBS $(10 \% \mathrm{~V} / \mathrm{V})$ and antibiotics $(100 \mathrm{U} / \mathrm{mL}$ penicillin and $100 \mathrm{mg} / \mathrm{mL}$ streptomycin) at $37^{\circ} \mathrm{C}$ in a $95: 5$ air $/ \mathrm{CO}_{2}$ humidified atmosphere.

\subsection{MTT Assays Performance}

Once grown, BT474, SKBR3 and HS5 cells were used to carry out MTT assays. For this purpose, they were seeded into 24 -well plates (12,000 cells/well) and incubated to allow attachment. The next day, culture medium in the wells of some plates was replaced by supplemented DMEM containing PBS (for the control), PDA NPs $(0.035$ and $0.042 \mathrm{mg} / \mathrm{mL})$, PTX ( 99.5 and $119.5 \mathrm{nM}$ ), Tmab (0.45 and $0.54 \mathrm{nM}$ ), PDA NPs•Tmab ( 0.035 and $0.042 \mathrm{mg} / \mathrm{mL})$, PDA NPs@PTX (0.035 and $0.042 \mathrm{mg} / \mathrm{mL})$ and PDA NPs•Tmab@PTX (0.035 and $0.042 \mathrm{mg} / \mathrm{mL})$ Culture medium in the wells of other plates was otherwise replaced by supplemented DMEM containing PBS, PDA NPs ( 0.035 and $0.042 \mathrm{mg} / \mathrm{mL}$ ), PTX (94.9 and $113.6 \mathrm{nM}$ ), Tmab (0.33 and $0.42 \mathrm{nM})$, PDA NPs@Tmab (0.035 and $0.042 \mathrm{mg} / \mathrm{mL})$, PDA NPs@PTX (0.035 and $0.042 \mathrm{mg} / \mathrm{mL}$ ) and PDA NPs@Tmab@PTX $(0.035$ and $0.042 \mathrm{mg} / \mathrm{mL})$. In both cases, cells were later incubated for 24,48 and $72 \mathrm{~h}$ and, after those times, a MTT solution $(5 \mathrm{mg} / \mathrm{mL}, 110 \mu \mathrm{L})$ was added to each well. Cell incubation was performed for an additional hour and formazan crystals were dissolved by adding DMSO (500 $\mu \mathrm{L} /$ well). The optical density value of each well was determined by applying a procedure that was previously set up to subtract PDA contribution to absorbance [17], which was measured in a microplate reader (Zetasizer Nano ZS90, Malvern Instruments Inc., Royston, UK). All data were obtained in triplicate.

\subsection{Live/death Fluorecence Staining of BT474 Cells}

In order to perform the first alive/death CLSM assays, BT474 cells were seeded $(12,000$ cells $/ \mathrm{mL})$ in glass-bottom dishes (Ibidi, Gräfelfing, Germany) and incubated for $24 \mathrm{~h}$. Subsequently, they were treated with or without PTX $(99.5 \mathrm{nM})$ or PDA NPs•Tmab@PTX $(0.035 \mathrm{mg} / \mathrm{mL})$ and incubated again. After 48 and $72 \mathrm{~h}$, cells were incubated with calcein $\mathrm{AM}(1 \mu \mathrm{M})$ and propidium iodide $(1 \mathrm{drop} / \mathrm{mL})$ fluorescent dyes for $15 \mathrm{~min}$. BT474 stained cells were imaged by CLSM (Leica TCS SP5, Leica Microsystems, L'Hospitalet de Llobregat, Barcelona, Spain) with an excitation/detection of 494/517 and 535/617 nm, respectively. The average number of living and dead cells after each type of treatment was determined from at least 5 different CLSM images.

\subsection{Flow Cytometry Analysis}

Flow cytometry data were obtained by an annexin $\mathrm{V}$ apoptosis detection kit with 7AAD according to the manufacturer's instructions (Immunostep, Salamanca, Spain). Briefly, BT474 cells were cultured in dishes $(12,000$ cells $/ \mathrm{mL})$, treated with or without PTX $(99.5 \mathrm{nM})$ and PDA NPs•Tmab@PTX $(0.035 \mathrm{mg} / \mathrm{mL})$ and incubated for $48 \mathrm{~h}$. After this time, cells were collected, centrifuged and resuspended in warm PBS. Then, they were washed by warm PBS twice and resuspended in annexin binding buffer $(1 \times)$ at a concentration of $10^{6}$ cells $/ \mathrm{mL}$. Annexin V $(5 \mu \mathrm{L})$ and 7AAD $(5 \mu \mathrm{L})$ were added to the cell samples $\left(10^{5}\right.$ cells $\left./ \mathrm{mL}, 100 \mu \mathrm{L}\right)$, and these were incubated at room temperature and in dark conditions for $15 \mathrm{~min}$. Stained cells were analyzed by flow cytometry (FACSAria ${ }^{\mathrm{TM}}$ III cytometer, BD Biosciences, San José, CA, USA), using FITC (green) for annexin V and PerCP (red) for 7AAD.

\subsection{Development of BT474 Spheroids and SEM Characterization}

BT474 MCTS were obtained by seeding the mentioned cells in Nunclon ${ }^{\mathrm{TM}}$ Sphera ${ }^{\mathrm{TM}}$ 96-well, U-shaped bottom microplates (Thermo Fisher Scientific, Eugene, OR, USA) $(10,000$ cells $/ \mathrm{mL})$ and allowing them to grow for 3 days. Then, they were treated with or 
without PTX (99.5 nM) or PDA NPs•Tmab@PTX $(0.035 \mathrm{mg} / \mathrm{mL})$, and 48 and $72 \mathrm{~h}$ later spheroids were characterized by SEM (JSM-IT500, Jeol, Tokyo, Japan). To prepare the samples, MCTS were carefully collected and sectioned, deposited on filter paper with poly-L-lysine and fixed overnight at $8{ }^{\circ} \mathrm{C}$ in PBS with glutaraldehyde $(25 \%)$. The next day, they were washed by PBS three times and after that, PBS with osmium (1\%) was added to the samples. One hour later, spheroids were washed by $\mathrm{H}_{2} \mathrm{O}(\mathrm{d})$ three times and were progressively dehydrated by a gradient concentration of acetone series. Critical point drying was achieved with $\mathrm{CO}_{2}$ and samples were observed by SEM after gold coating.

\subsection{Live/Death Fluorecence Staining and Cell-Counting of BT474 Spheroids}

Live/death CLSM assays with BT474 spheroids were performed in the same way as those with the monolayer cell cultures, simply developing them on the microplates mentioned in the previous point and depositing them once grown in glass-bottom dishes.

Otherwise, to assess BT474 spheroid viability by cell-counting, these grown 3D cellular spheres were kept on the U-shaped bottom microplates, where they were treated with or without PTX $(99.5 \mathrm{nM})$, bare PDA NPs $(0.035 \mathrm{mg} / \mathrm{mL})$ or PDA NPs•Tmab@PTX $(0.035 \mathrm{mg} / \mathrm{mL})$. After $48 \mathrm{~h}$, MCTS were washed by warm PBS, disaggregated with trypsin, collected in DMEM and centrifuged. Cells were resuspended in supplemented DMEM and their viability was determined (InvitrogenCountess II FL Automated Cell Counter, ThermoFisher Scientific) after mixing them with trypan blue. Data were again obtained in triplicate.

\subsection{Statistical Analysis}

Data regarding the size (DLS) and zeta potential values of PDA NPs were the average \pm SEM of three different measurements. Data concerning MTT assays were analyzed using an unpaired two-tailed Student $t$-test. $p$-values less than 0.05 were considered to be statistically significant. Displayed viability results were the average \pm SEM of three replicates per treatment condition.

\section{Conclusions}

In conclusion, this report has presented the synthesis of PDA NPs that could act as targeted nanocarriers for PTX and Tmab for the treatment of HER2+ breast tumors. The combination of this taxane and this anti-HER2 antibody is extensively used in the clinical setting as adjuvant therapy and, given its great results, several DDS transporting PTX and Tmab have already been developed. However, neither the taxane or the anti-HER2 antibody have been loaded to PDA NPs to date, and the resulting drug delivery NPs have been shown to reduce normal cell viability rate to a lesser extent than the parent drug and to have a very remarkable antitumor activity in vitro, not only in HER2+ conventional cell cultures, but also in breast tumor spheroids.

Thus, if Tmab-PTX loaded PDA NPs would also yield good results in vivo in the future, they may constitute an excellent approach to overcome the many disadvantages that the current adjuvant treatment of Tmab and PTX entails, mainly related to their toxicity and the apparition of resistances.

Supplementary Materials: The following are available online at https:/ /www.mdpi.com/article/10 .3390 / cancers13112526/s1, Figure S1: survival rates of BT474, SKBR3 and HS5 cells after treatment bare PDA NPs, PDA NPs-Tmab, PDA NPs@PTX and PDA NPs-Tmab@PTX, as well as with concentrations of free Tmab and PTX similar to those loaded to PDA NPs; Figure S2: phase-contrast images of BT474 spheroids $48 \mathrm{~h}$ after treatment with or without PDA NPs•Tmab@PTX.

Author Contributions: Conceptualization, C.N., M.A.V. and E.M.d.V.; methodology, C.N. and M.A.V.; software, C.N. and M.A.V.; validation, C.N. and M.A.V.; investigation, C.N., M.A.V. and E.M.d.V.; writing-original draft preparation, C.N.; writing-review and editing, C.N., M.A.V. and E.M.d.V.; supervision, M.A.V. and E.M.d.V.; project administration, E.M.d.V.; funding acquisition, E.M.d.V. All authors have read and agreed to the published version of the manuscript. 
Funding: This research was funded by the Spanish Ministry of Sciences, Innovation and Universities, grant number PID2019-1088994R-B100.

Institutional Review Board Statement: Not applicable.

Informed Consent Statement: Not applicable.

Data Availability Statement: The data that support the findings of this study are available from the corresponding authors upon reasonable request.

Acknowledgments: C.N. thanks Junta de Castilla y León for her predoctoral fellowship, and all the authors thank the Electron Microscopy Facilities (NUCLEUS) of the University of Salamanca for the SEM images.

Conflicts of Interest: The authors declare no conflict of interest.

$\begin{array}{ll}\text { Abbreviations } & \\ \text { BC } & \text { Breast cancer } \\ \text { CLSM } & \text { Confocal laser scanning microscopy } \\ \text { DDS } & \text { Drug delivery system } \\ \text { DLS } & \text { Dynamic light scattering } \\ \text { HER2 } & \text { Human epidermal growth factor receptor 2 } \\ \text { HER2+ } & \text { Human epidermal growth factor receptor 2-positive } \\ \text { mAb } & \text { Monoclonal antibody } \\ \text { MCTS } & \text { Multicellular tumor spheroid } \\ \text { NPs } & \text { Nanoparticles } \\ \text { PDA } & \text { Polydopamine } \\ \text { PDA NPs@PTX } & \text { Polydopamine nanoparticles loaded with paclitaxel through adsorption } \\ \text { PDA NPs•Tmab } & \text { Polydopamine nanoparticles covalently loaded with trastuzumab } \\ \text { PDA NPs@Tmab } & \text { Polydopamine nanoparticles loaded with trastuzumab } \\ & \text { through adsorption } \\ \text { PDA NPs•Tmab@PTX } & \text { Polydopamine nanoparticles covalently loaded with trastuzumab and } \\ & \text { with adsorbed paclitaxel } \\ \text { PDA NPs@Tmab@PTX } & \text { Polydopamine nanoparticles loaded with both trastuzumab and } \\ \text { PTX } & \text { paclitaxel through adsorption } \\ \text { ROS } & \text { Paclitaxel } \\ \text { SEM } & \text { Reactive oxygen species } \\ \text { TEM } & \text { Scanning electron microscopy } \\ \text { TK } & \text { Transmission electron microscopy } \\ \text { Tmab } & \text { Tyrosine kinase } \\ \text { 2-PrOH } & \text { Trastuzumab } \\ & \text { 2-propanol }\end{array}$

\section{References}

1. Global Cancer Observatory. Available online: https://gco.iarc.fr/today/data/factsheets/cancers/20-Breast-fact-sheet.pdf (accessed on 8 April 2021).

2. Dieci, M.V.; Miglietta, F.; Griguolo, G.; Guarneri, V. Biomarkers for HER2-positive metastatic breast cancer: Beyond hormone receptors. Cancer Treat. Rev. 2020, 88, 102064. [CrossRef] [PubMed]

3. Elseginy, S.A.; Hamdy, R.; Menon, V.; Almehdi, A.M.; El-Awady, R.; Soliman, S.S.M. Design, synthesis, and computational validation of novel compounds selectively targeting HER2-expressing breast cancer. Bioorg. Med. Chem. Lett. 2020, $30,127658$. [CrossRef] [PubMed]

4. Dhritlahre, R.K.; Saneja, A. Recent advances in HER2-targeted delivery for cancer therapy. Drug Discov. Today 2020. In press. [CrossRef]

5. Nieto, C.; Vega, M.A.; Martín del Valle, E.M. Trastuzumab: More than a guide in HER2-positive cancer nanomedicine. Nanomaterials 2020, 10, 1674. [CrossRef]

6. Stewart, P.; Blanchette, P.; Shah, P.S.; Ye, X.Y.; Boldt, R.G.; Fernandes, R.; Vanderberg, T.; Rhapael, J. Do all patients with HER2 positive breast cancer require one year of adjuvant trastuzumab? A systematic review and meta-analysis. Breast 2020, 54, 203-210. [CrossRef] [PubMed] 
7. De Sanctis, R.; Giordano, L.; D’Antonio, F.; Agostinetto, E.; Marinello, A.; Guiducci, D.; Masci, G.; Losurdo, A.; Zuradelli, M.; Torrisi, R.; et al. Clinical predictors of cardiac toxicity in HER2-positive early breast cancer patients treated with adjuvant s.c. versus i.v. trastuzumab. Breast 2021, 57, 80-85. [CrossRef] [PubMed]

8. Plosker, G.L.; Keam, S.J. Trastuzumab. Drugs 2006, 66, 449-475. [CrossRef] [PubMed]

9. Nahta, R.; Esteva, F.J. HER2 therapy: Molecular mechanisms of trastuzumab resistance. Breast Cancer Res. 2006, 8, 215. [CrossRef]

10. Marques, A.C.; Costa, P.J.; Velho, S.; Amaral, M.H. Functionalizing nanoparticles with cancer-targeting antibodies: A comparison of strategies. J. Control. Release 2020, 320, 180-200. [CrossRef] [PubMed]

11. Wicki, A.; Witzigmann, D.; Balasubramanian, V.; Huwyler, J. Nanomedicine in cancer therapy: Challenges, opportunities and clinical applications. J. Control. Release 2015, 200, 138-157. [CrossRef]

12. Slamon, D.; Eiermann, W.; Robert, N.; Pienkowski, T.; Martín, M.; Press, M.; Mackey, J.; Glaspy, J.; Chan, A.; Pawlicki, M.; et al. Adjuvant trastuzumab in HER2-positive breast cancer. N. Engl. J. Med. 2011, 365, 1273-1283. [CrossRef]

13. Zhao, L.; Bi, D.; Qi, X.; Guo, Y.; Yue, F.; Wang, X.; Han, M. Polydopamine based surface modification of paclitaxel nanoparticles for osteosarcoma targeted therapy. Nanotechnology 2019, 30, 255101. [CrossRef] [PubMed]

14. Samaan, T.M.A.; Samec, M.; Liskova, A.; Kubatka, P.; Brïsselberg, D. Paclitaxel's mechanistic and clinical effects on breast cancer. Biomolecules 2019, 9, 789. [CrossRef] [PubMed]

15. Raj, S.; Khurana, S.; Choudhari, R.; Kesari, K.K.; Kamal, M.A.; Garg, N.; Ruokolainen, J.; Das, B.C.; Kumar, D. Specific targeting cancer cells with nanoparticles and drug delivery in cancer therapy. Semin. Cancer Biol. 2019. In Press. [CrossRef] [PubMed]

16. Nieto, C.; Centa, A.; Rodríguez-Rodríguez, J.A.; Pandiella, A.; Martín del Valle, E.M. Paclitaxel-trastuzumab mixed nanovehicle to target HER2-overexpressing tumors. Nanomaterials 2019, 9, 948. [CrossRef] [PubMed]

17. Nieto, C.; Vega, M.A.; Marcelo, G.; Martín del Valle, E.M. Polydopamine nanoparticles kill cancer cells. RSC Adv. 2018, 8, 36201-36208. [CrossRef]

18. Ambekar, R.S.; Kandasubramanian, B. A polydopamine-based platform for anti-cancer drug delivery. Biomater. Sci. 2019, 7, 1776-1793. [CrossRef]

19. Wang, W.; Tang, Z.; Zhang, Y.; Wang, Q.; Liang, Z.; Zeng, X. Mussel-inspired polydopamine: The bridge for targeting drug delivery system and synergistic cancer treatment. Macromol. Biosci. 2020, 20, 200022. [CrossRef] [PubMed]

20. Wu, H.; Hu, H.; Wan, J.; Li, Y.; Wu, Y.; Tang, Y.; Xiao, C.; Xu, H.; Yang, X.; Li, Z. Hydroxyethyl starch stabilized polydopamine nanoparticles for cancer chemotherapy. Chem. Eng. J. 2018, 349, 129-145. [CrossRef]

21. Hao, Y.-N.; Zheng, A.-Q.; Guo, T.-T.; Shu, Y.; Wang, J.-H.; Johnson, O.; Chen, W. Glutathione triggered degradation of polydopamine to facilitate controlled drug release for synergic combinational cancer treatment. J. Mater. Chem. B 2019, 7, 6742-6750. [CrossRef]

22. Nieto, C.; Vega, M.A.; Enrique, J.; Marcelo, G.; Martín del Valle, E.M. Size matters in the cytotoxicity of polydopamine nanoparticles in different types of tumors. Cancers 2019, 11, 1679. [CrossRef]

23. Nieto, C.; Marcelo, G.; Vega, M.; Martín del Valle, E.M. Antineoplastic behaviour of polydopamine nanoparticles prepared in different water/alcohol media. Colloids Surf. B Biointerfaces 2021, 199, 111506. [CrossRef] [PubMed]

24. Vega, M.A.; Nieto, C.; Marcelo, G.; Martín del Valle, E.M. Cytotoxicity of paramagnetic cations-loaded polydopamine nanoparticles. Colloids Surf. B Biointerfaces 2018, 167, 284-290. [CrossRef] [PubMed]

25. Lizunova, A.A.; Loshkarev, A.A.; Tokunov, Y.M.; Ivanov, U.V. Comparison of the results of measurements of the sizes of nanoparticles in stable colloidal solutions by the methods of acoustic spectroscopy, dynamic light scattering, and transmission electron microscopy. Meas. Teach. 2017, 59, 1151-1155. [CrossRef]

26. Madison, S.A.; Carnali, J.O. pH optimization of amidation via carbodiimides. Ind. Eng. Chem. Res. 2013, 52, 13547-13555. [CrossRef]

27. Park, J.; Kim, H.-D.; Lee, S.-H.; Kwak, C.-H.; Chang, Y.-C.; Lee, Y.-C.; Chung, T.-W.; Magae, J.; Kim, C.-H. Ascochlorin induces caspase-independent necroptosis in LSP-stimulated RAW 264.7 macrophages. J. Ethnopharmacol. 2019, 39, 111898. [CrossRef] [PubMed]

28. Ishiguro, T.; Ohata, H.; Sato, A.; Yamawaki, K.; Enomoto, T.; Okamoto, K. Tumor-derived spheroids: Relevance to cancer stem cells and clinical applications. Cancer Sci. 2017, 108, 283-289. [CrossRef] [PubMed]

29. Weiswald, L.-B.; Bellet, D.; Dangles-Marie, V. Spherical cancer models in tumour biology. Neoplasia 2015, 17, 1-15. [CrossRef]

30. Mollo, V.; Scognamiglio, P.; Marino, A.; Ciofani, G.; Santoro, F. Probing the ultrastructure of spheroids and their uptake of magnetic nanoparticles by FIB-SEM. Adv. Mater. Technol. 2020, 5, 1900687. [CrossRef]

31. Nath, S.; Devi, G.R. Three-dimensional culture systems in cancer research: Focus on tumor spheroid model. Pharmacol. Ther. 2016, 163, 94-108. [CrossRef]

32. Castro, J.S.; Tapia, L.V.; Silveyra, R.A.; Martinez, C.A.; Deymier, P.A. Negative impact of paclitaxel crystallization in hydrogels and novel approaches for anticancer drug delivery systems. In Current Cancer Treatment: Novel Beyond Conventional Approaches; Ozdemir, O., Ed.; IntechOpen Limited: Rijeka, Croatia, 2011; pp. 767-782.

33. Tchoryk, A.; Taresco, V.; Argent, R.H.; Ashford, M.; Gellert, P.R.; Stolnik, S.; Grabowska, A.; Garnett, M.C. Penetration and uptake of nanoparticles in 3D tumor spheroids. Bioconjug. Chem. 2019, 30, 1371-1384. [CrossRef] [PubMed]

34. Lee, A.L.Z.; Wang, Y.; Cheng, H.Y.; Pervaiz, S.; Yang, Y.Y. The co-delivery of paclitaxel and Herceptin using cationic micellar nanoparticles. Biomaterials 2009, 30, 919-927. [CrossRef] 
35. Chang, D.; Gao, Y.; Wang, L.; Liu, G.; Chen, Y.; Wang, T.; Tao, W.; Mei, L.; Huang, L.; Zeng, X. Polydopamine-based surface modification of mesoporous silica nanoparticles as $\mathrm{pH}$-sensitive drug delivery vehicles for cancer therapy. J. Colloids Interface Sci. 2016, 463, 279-287. [CrossRef] [PubMed]

36. Bi, D.; Zhao, L.; Yu, R.; Li, H.; Guo, Y.; Wang, X.; Han, M. Surface modification doxorubicin-loaded nanoparticles based on polydopamine with $\mathrm{pH}$-sensitive property for tumor targeting therapy. Drug Deliv. 2018, 25, 564-575. [CrossRef] [PubMed]

37. Nieto, C.; Vega, M.A.; Martín del Valle, E.M. Tailored-made polydopamine nanoparticles to induce ferroptosis in breast cancer cells in combination with chemotherapy. Int. J. Mol. Sci. 2021, 22, 3161. [CrossRef]

38. Yousefpour, P.; Atyabi, F.; Vasheghani-Farahani, E.; Movahedi, A.-A.M.; Dinarvand, R. Targeted delivery of doxorubicin-utilizing chitosan nanoparticles surface-functionalized with anti-HER2 trastuzumab. Int. J. Nanomed. 2011, 6, 1977-1990. 